\title{
Bioequivalence Study of Two Hydrochlorothiazide Formulations after Oral Administration in Healthy Chilean Volunteers
}

\author{
Fierro Humberto ${ }^{1}$, Rojas Romina ${ }^{1}$, Yáñez Sebastian ${ }^{2}$, Acuña Paola ${ }^{2}$, Rosales Katherine ${ }^{2}$, Yevenes Paulina ${ }^{3}$, \\ Yzoard Marcia $^{3}$, Tapia Claudia ${ }^{3}$, Rivas Violeta ${ }^{3}$, Caba Alvaro ${ }^{1}$, Copaja Miguel ${ }^{2}$ and Jorge Fuentealba ${ }^{1,4}$ \\ 1. Center for Advanced Research on Biomedicine (CIAB-UdeC), University of Concepción, Concepción 4070038, Chile \\ 2. Area Health and Safety, AGQ, Santiago 8590829, Chile \\ 3. Regional Hospital Guillermo Grant Benavente, Concepción 4070038, Chile \\ 4. Laboratory of Screening of Neuroactive Compounds, Department of Physiology Faculty of Biological Sciences, University of \\ Concepcion, Concepción 4070038, Chile
}

\begin{abstract}
The study was carried out in healthy Chilean volunteers in order to compare the pharmacokinetics (rate and extent absorption) of two commercial oral formulations of $50 \mathrm{mg}$ hydrochlorothiazide. Thirty nine subjects were administered hydrochlorothiazide tablets of test $(T)$ and reference $(R)$ formulation in a single blind, randomized, fasting, $2 \times 2$ crossover study, seven washout days. Blood samples were taken during a $48 \mathrm{~h}$ period after drug administration. Plasma concentrations were quantified by HPLC-MS/MS. The primary parameters $\log$-transformed $C_{\max }$ (maximum plasma concentration), $A U C_{0-t}$ and $A U C_{0-\infty}$ (area under the plasma concentration-time curve from zero to the last time and zero to infinity) were tested for bioequivalence considering the ratios of geometric means (test/reference); whereas $t_{\max }$ (the time of maximum plasma concentration) was analyzed nonparametrically. The $90 \%$ confidence intervals for the geometric mean values of test/reference ratios for $C_{\max }, A U C_{0-t}$ and $A U C_{0-\infty}$ were $95.37 \%, 93.59 \%$ and $96.34 \%$, respectively, and were located within the bioequivalence acceptance range of $80 \sim 125 \%$, as were $t_{\max }$ and elimination constants. Together, we conclude that the test formulation of the hydrochlorothiazide $50 \mathrm{mg}$ tablet is bioequivalent to the reference product and suitable for generic prescription.
\end{abstract}

Key words: Bioequivalence, hydrochlorothiazide, interchangeable.

\section{Introduction}

The term thiazide diuretics is used here to refer to all members of the class of $\mathrm{Na}^{+} / \mathrm{Cl}^{-}$symporter inhibitors [1]. Hydrochlorothiazide (6-chloro-3,4-dihydro-2H-1,2,4-enzothiadiazine-7-sulf onamide 1,1-dioxide) is a potent thiazide diuretic that enhances natriuresis which leads to a reduction in plasma volume and cardiac output [2]. Thiazide diuretics are used for the treatment of edema associated with heart (congestive heart failure), liver (hepatic cirrhosis), and renal (nephrotic syndrome, chronic

Corresponding author: Jorge Patricio Fuentealba Arcos, Ph.D., research fields: neurosciences, Alzheimer disease, pharmacology and pharmacokinetics. renal failure, and acute glomerulonephritis) disease $[1,2]$. Therefore, hydrochlorothiazide is widely used alone or in combination with other antihypertensive drugs for the treatment of hypertension and congestive heart failure [3]. This drug has been used for a long time and is regarded as a highly safe medication although some common and frequent adverse effects are hypokalemia, hyperuricaemia, decreased glucose tolerance, hyperlipidaemia, impotence and lethargy [1,2].

Hydrochlorothiazide is readily absorbed from the gastrointestinal tract and its absolute bioavailability following oral administration is approximately $60 \sim 80 \%$ of the dose $[1,3]$. The peak plasma concentration is 
achieved around $2 \mathrm{~h}$ after oral administration and the elimination half-life is approximately $10 \mathrm{~h} \mathrm{[4].} \mathrm{The}$ diuretic effect persists $6 \sim 12 \mathrm{~h}$ and the hypotensive effect persists for up to one week after therapy withdrawal [4]. The bioavailable dose fraction is excreted almost completely in the urine as unchanged drug, and $50 \sim 70 \%$ of the oral dose is excreted during the next $24 \mathrm{~h}$ after oral administration [3, 4].

Thiazide diuretics were the first tolerated efficient antihypertensive drugs that significantly reduced cardiovascular morbidity and mortality in placebo-controlled clinical studies [5]. Today, these drugs are still considered a fundamental therapeutic tool in the clinical arsenal for treatment of hypertensive patients. However, a high correlation between oral dose and plasma concentration is important to guarantee the quality of the medication and ensure an optimal therapeutic response [5].

Generic drug medication is fundamental for the general population, especially those in the low economic sector of the public health system, in order to have access to quality drugs with lower costs [6]. In most cases, the innovator formulation is usually too expensive for the majority of patients. Therefore, the use of certified generic drugs is essential for the population to have equitable access to treatment with the same quality of innovators, but with reduced costs.

Interchangeability of drugs is established by bioequivalence studies comparing the plasma concentration versus time curves for the generic and the innovator formulations. They are considered bioequivalent when the rate and extent of bioavailability of the active ingredient in the two products are not significantly different under suitable test conditions [6, 7].

The objective of this study was to perform a bioequivalence analyses of two $50 \mathrm{mg}$ tablet formulations of hydrochlorothiazide after a single oral administration under fasting conditions in healthy volunteers. In addition, our study provides the Chilean population with a new certified low cost therapeutic alternative; and also characterizes the pharmacokinetic parameters of Chilean subjects to this type of drug.

\section{Materials and Methods}

Hydrochlorothiazide in plasma samples were analyzed using a validated method involving HPLC (Agilent 1200 Series, Agilent Technologies, Waldbronn, Germany) with MS/MS detection (API 4000, MDS Sciex, Ontario, Canada) in AGQ Labs, Santiago, Chile. The developed method was validated with respect to the following validation parameters: selectivity, linearity and linear working range, limits of detection and quantitation, recovery, accuracy, precision, and stability. The validation follows the FDA (US Food and Drug Administration) guideline for bioanalytical methods. The stability evaluation showed that Hydrochlorothiazide was stable in plasma at least 40 days when stored at $-75{ }^{\circ} \mathrm{C}$. Hydrochlorothiazide and the internal standard (Losartan) were isolated from $0.3 \mathrm{~mL}$ plasma samples by protein precipitation using acetonitrile. Aliquots of plasma extracts $(10 \mu \mathrm{L})$ were subjected to chromatographic separation using an Agilent Poroshell 120 EC-C18 column $(2.7$ um $3.0 \times 50 \mathrm{~mm})$ thermostated at $40{ }^{\circ} \mathrm{C}$. The mobile phase was (1) water with $0.2 \%$ acetic acid, ammonium acetate $10 \mathrm{mM}$ and (2) acetonitrile HPLC-grade (B/A; 60/40 v/v\%). The separation was performed under isocratic conditions with a constant flow rate of $0.3 \mathrm{~mL} / \mathrm{min}$. LC-MS/MS experimental conditions utilized the multiple reaction monitoring, and detection of hydrochlorothiazide and internal standard (Losartan) was performed in the negative ESI mode for their respective $[\mathrm{M}-\mathrm{H}]^{-}$ions. Calibration curves were linear in the range of $10 \sim 450 \mathrm{ng} / \mathrm{mL}$ with a coefficient of correlation of $r \geq 0.9969$ and a lower limit of quantification of $10 \mathrm{ng} / \mathrm{mL}$. Recovery of hydrochlorothiazide ranged from $94.5 \%$ to $100 \%$. Inter-assay precision (expressed as a coefficient of variation) was $\leq 9.29 \%$; and inter-assay accuracy was $\leq 9.14 \%$. All solvents were of HPLC (high-pressure 
liquid chromatography) grade.

\subsection{Subjects}

The study sample size was calculated based on a

Table 1 Demographic data of healthy volunteers participant on the study.

\begin{tabular}{|c|c|c|c|c|}
\hline Volunteer & Sex & Age (years) & $\mathrm{BMC}$ & Sequence \\
\hline 1 & M & 25 & 21.6 & $\mathrm{AB}$ \\
\hline 2 & M & 25 & 25.1 & BA \\
\hline 3 & M & 20 & 29.6 & $\mathrm{AB}$ \\
\hline 4 & $\mathrm{~F}$ & 27 & 27 & $\mathrm{BA}$ \\
\hline 5 & $\mathrm{~F}$ & 24 & 20.8 & $\mathrm{AB}$ \\
\hline 6 & $\mathrm{~F}$ & 20 & 21.1 & BA \\
\hline 9 & M & 27 & 26.9 & BA \\
\hline 10 & $\mathrm{~F}$ & 24 & 22.4 & $\mathrm{AB}$ \\
\hline 12 & $\mathrm{~F}$ & 24 & 23.4 & $\mathrm{AB}$ \\
\hline 14 & $\mathrm{~F}$ & 24 & 20.8 & $\mathrm{AB}$ \\
\hline 16 & M & 23 & 25.1 & BA \\
\hline 17 & M & 37 & 26 & $\mathrm{AB}$ \\
\hline 18 & $\mathrm{~F}$ & 24 & 23.4 & $\mathrm{AB}$ \\
\hline 19 & M & 20 & 23.4 & $\mathrm{AB}$ \\
\hline 20 & M & 21 & 27.5 & BA \\
\hline 21 & M & 20 & 24.1 & $\mathrm{AB}$ \\
\hline 22 & M & 23 & 25.4 & BA \\
\hline 23 & M & 24 & 22.2 & $\mathrm{AB}$ \\
\hline 24 & M & 21 & 23.7 & BA \\
\hline 25 & $\mathrm{~F}$ & 21 & 23.4 & $\mathrm{AB}$ \\
\hline 26 & M & 23 & 29.1 & BA \\
\hline 27 & $\mathrm{~F}$ & 23 & 18.3 & $\mathrm{AB}$ \\
\hline 28 & M & 22 & 20.9 & BA \\
\hline 30 & M & 18 & 19.6 & BA \\
\hline 32 & $\mathrm{~F}$ & 26 & 21.1 & $\mathrm{AB}$ \\
\hline 33 & $\mathrm{~F}$ & 19 & 18.6 & BA \\
\hline 34 & M & 26 & 29.4 & $\mathrm{AB}$ \\
\hline 35 & $\mathrm{~F}$ & 19 & 26 & $\mathrm{BA}$ \\
\hline 36 & M & 19 & 19.5 & $\mathrm{AB}$ \\
\hline 37 & $\mathrm{~F}$ & 23 & 19.3 & $\mathrm{AB}$ \\
\hline 38 & M & 23 & 23.1 & BA \\
\hline 39 & $\mathrm{~F}$ & 23 & 19.5 & $\mathrm{AB}$ \\
\hline 40 & $\mathrm{~F}$ & 23 & 22.8 & BA \\
\hline 41 & M & 22 & 26.5 & $\mathrm{AB}$ \\
\hline 44 & M & 21 & 20.6 & $\mathrm{AB}$ \\
\hline 45 & M & 27 & 26.2 & BA \\
\hline 46 & M & 21 & 22.6 & $\mathrm{AB}$ \\
\hline 47 & M & 22 & 21.6 & BA \\
\hline 48 & M & 20 & 23.5 & $\mathrm{AB}$ \\
\hline Mean \pm SD & & $22.9 \pm 3.3$ & $23.4 \pm 3$ & \\
\hline
\end{tabular}

F: female; M: male; SD: standard deviation; AB: test-reference; BA: reference-test. within-subject $\mathrm{CV} \%$ of $A U C_{0-\infty}$ and $C_{\max }[8,9]$. Forty-eight healthy male and female adult volunteers were enrolled in this study, which was performed in the Guillermo Grant Benavente Hospital, Concepcion, Chile. The age range of the subjects was 18 30 years, with a weight range of 52 89 kg and a height range of 154 185 cm (Table 1). All subjects gave written informed consent and the protocol form was approved prior to the start of the study by the Scientific and Ethic Committee of the Health Service of Concepcion (CEC), and National Public Health Authority (Instituto de Salud Pública de Chile) of Chile. The study was conducted in accordance with Good Clinical Practice guidelines and according to the Revised Declaration of Helsinki [10].

\subsection{Clinical Protocol}

The study was a single-dose, 2-way randomized crossover design with a one week washout period between the doses. The subjects were instructed to abstain from taking any medication for at least one week before and during the study period. No subject was alcoholic or a smoker and no alcohol was allowed $48 \mathrm{~h}$ before each study period and until after the last sample in each period had been collected. The subjects were also not allowed to drink grapefruit juice or beverages or food containing xanthines, such as tea, coffee and cola. Screening at the beginning of the study included physical examination, medical history, laboratory safety tests (hemoglobin, hematocrit, total and differential white cell count, creatinine, alkaline phosphatase, Alanin Trans Aminase/ALT, total bilirubin, albumin and total protein, routine urinalysis) and vital signs. During the enrollment process and each of the clinical phases, all volunteers were tested for drug abuse consumption. All clinical laboratory tests were performed at Guillermo Grant Benavente Hospital, Concepcion, Chile.

The subjects fasted for $10 \mathrm{~h}$ prior to drug administration. On the morning of the study phase, volunteers were given a single dose of reference 
formulation (Clorana ${ }^{\circledR}$, Sanofi Adventis Laboratory), or test formulation (Hydrochlorothiazide ${ }^{\circledR}$, Laboratory Chile) of a $50 \mathrm{mg}$ hydrochlorothiazide tablet. General instructions were provided to all volunteers. Briefly, no food was allowed for $2 \mathrm{~h}$ after dosing, food was given according to the time schedule and nutritionist recommendations, and the volunteers were instructed to stay in bed for $5 \mathrm{~h}$ after drug administration. Blood pressure, pulse, and adverse events were monitored and recorded in the CRF (Case Report Form) by our clinical staff. The samples for $24 \mathrm{~h}, 36 \mathrm{~h}$ and $48 \mathrm{~h}$ were taken under ambulatory conditions.

\subsection{Drug Administration and Sample Collection}

The drugs (test or reference) were given to the volunteers with $250 \mathrm{~mL}$ of water. Blood sample volumes were $5.0 \mathrm{~mL}$ at each sampling time $(0.5,1,1.5$, $2,2.5,3,3.5,4,4.5,5,6,8,12,24,36$ and $48 \mathrm{~h}$ after dosing). Samples were centrifuged at $4,000 \mathrm{~g}$ for $5 \mathrm{~min}$ immediately after collection, and the plasma was stored at $-20{ }^{\circ} \mathrm{C}$ until assay. After seven days (washout period), the study was repeated under the same conditions to complete the crossover design. Counter samples were stored at $-80^{\circ} \mathrm{C}$ in the Physiology Department at the University of Concepcion.

\subsection{Pharmacokinetic Analysis}

Pharmacokinetic parameters were determined and calculated using a Winnolin version 6.3 computer program. The data was described by arithmetic mean, standard deviation and $95 \%$ confidence intervals. The elimination rate constant $(\mathrm{Ke})$ was obtained from the least-square fitted terminal log-linear portion of the plasma concentration-time profile. The hydrochlorothiazide $C_{\max }$ and the corresponding $T_{\max }$ were determined by the individual drug serum concentration-time profiles. The elimination half-life $\left(T_{1 / 2}\right)$ was calculated as $0.693 / \mathrm{Ke}$. The area under the curve to the last measurable concentration $\left(A U C_{0-t)}\right.$ was calculated by the linear trapezoidal rule. The area under the curve extrapolated to infinity $\left(A U C_{0-\infty}\right)$ was calculated as $A U C_{0-t}+C_{t} / \mathrm{Ke}$, where $C_{t}$ was the last measurable concentration. Bioequivalence was accepted if the calculated $90 \%$ confidence intervals were within $0.80 \sim 1.25$ for $A U C_{0-t}$ and $C_{\max }$. Analysis of $C_{\text {max }}$ and $A U C$ was carried out by ANOVA (analysis of variance) using Prism 5.0.1, GraphPad Software Inc. To improve the normality of the distribution, logarithmic transformation was applied prior to the analyses. The $90 \%$ CIs (confidence intervals) were constructed for the ratio of geometric means of tested and reference products and were compared to the reference intervals $(0.8 \sim 1.25)$ as suggested by the FDA [11].

\section{Results}

The analytical method to quantify hydrochlorothiazide was developed in order to use a $0.3 \mathrm{~mL}$ of plasma sample, and losartan was used as the internal standard. The calibration curve for hydrochlorothiazide showed linearity between 10 and $450 \mathrm{ng} / \mathrm{mL}$ (Fig. 1a), with a confidence interval of $95 \%$ and two-freedom degree. Thus, we had optimal conditions for the quantification of all samples obtained from the clinical stages. Each sample was injected three times to make the quantification of each time of sampling. Additionally, all fortification levels showed a coefficient of variations under $15 \%$ for all parameters considered in this methodological validation: recovery, precision (intra and between days),

Table 2 Bioequivalence assessment summary.

\begin{tabular}{lcll}
\hline Parameter $^{\mathrm{a}}$ & Test/reference (\%) & Reference & Test \\
\hline$C_{\max }(\mathrm{ng} / \mathrm{mL})$ & 95.37 & $280.81 \pm 1.05$ & $267.81 \pm 1.05$ \\
$A U C_{0-t}\left(\mathrm{~h}^{*} \mathrm{ng} / \mathrm{mL}\right)$ & 95.59 & $1669.99 \pm 1$ & $1563.08 \pm 1.05$ \\
$A U C_{0-\infty}\left(\mathrm{h}^{*} \mathrm{ng} / \mathrm{mL}\right)$ & 96.34 & $1730.39 \pm 1.05$ & $1667.10 \pm 1.05$ \\
$T_{\max }(\mathrm{h})$ & 105.98 & $2.07 \pm 1.06$ & $2.19 \pm 1.06$ \\
\hline
\end{tabular}

\footnotetext{
${ }^{\text {a }}$ Pharmacokinetic parameters are given as mean (SEM).
} 


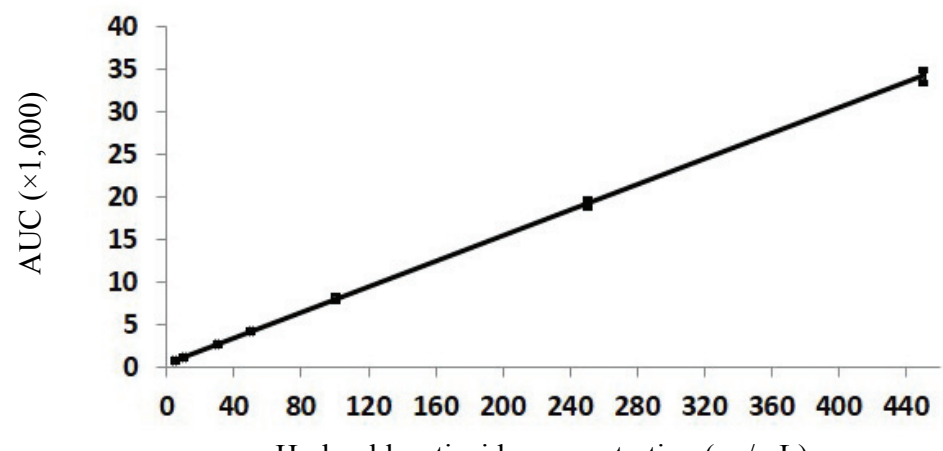

(a)

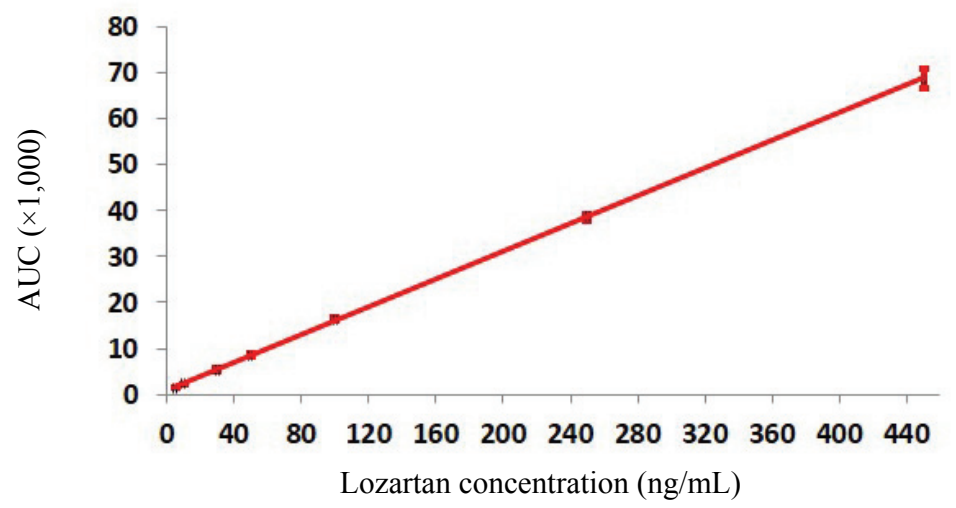

(b)

Fig. 1 Calibration curve of hydrochrolothiazide: (a) linear regression of the calibration curve for hydrochlorothiazide between 10 and $450 \mathrm{ng} / \mathrm{mL}$ at injection time for each point; (b) linear regression of the calibration curve for losartan, used as an internal standard, at the same concentration range as the analyte $(n=3$, for each point).

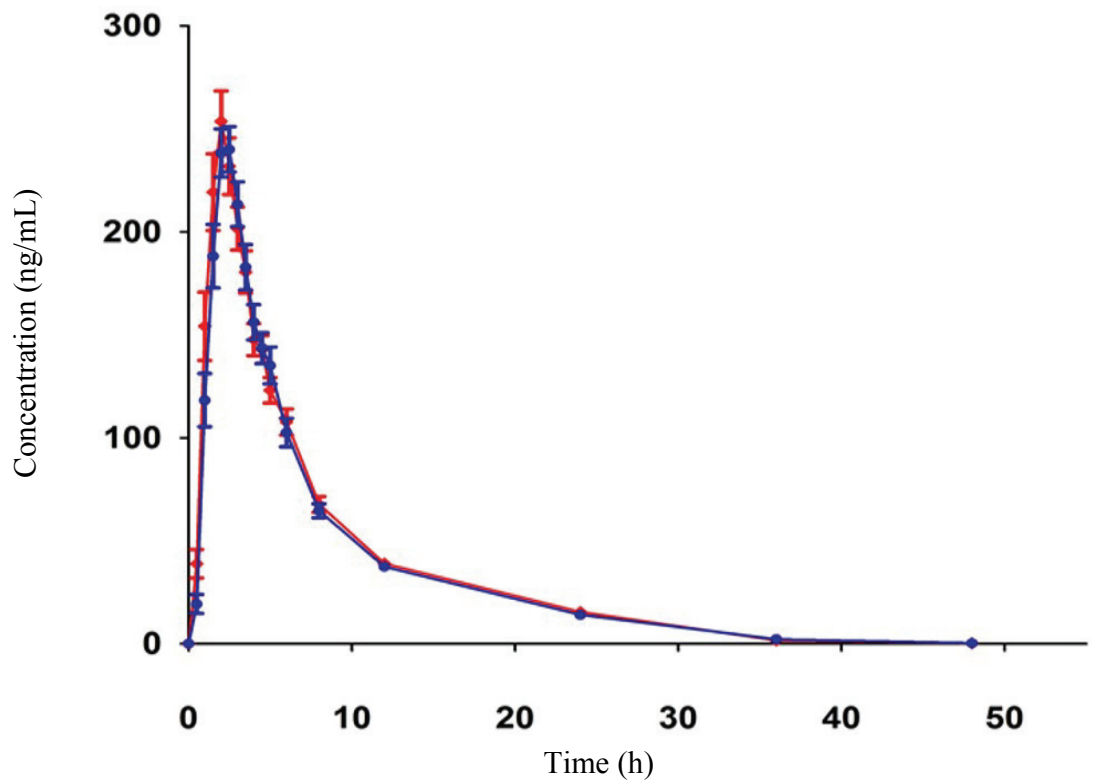

Fig. 2 Linear profile of the mean concentration versus time curve and variations in plasma concentration during the sampling time. The values represent the mean \pm SEM of 39 volunteers monitored for $48 \mathrm{~h}$. The blue line corresponds to the pharmacokinetics of the reference formulation, and the red line corresponds to the pharmacokinetics of the test formulation. No statistical differences were found $(n=39)$. 
exactitude (intra and between days), and uncertainty (Table 2). In parallel, the internal standard losartan showed linearity in the same range of concentrations similar to the analyte (Fig. 1b).

Thirty-nine healthy Chilean volunteers (25 men, 14 women) completed the study. The general demographic volunteer information and sequence are summarized in Table 1. The mean participant age was 22.9 years old, and the mean BCM (body corporal mass) was around 23. No serious or unexpected adverse events were observed; however, nine volunteers were excluded during the study due to difficulties with their venous access, personal reasons, or desertion, and were replaced. The safety profile was similar for the test and reference formulations and it was consistent with the summary of the product characteristics [5]. The most frequently observed adverse event was hypotension followed by dizziness and headache.

Variations in plasma concentration during the sampling time after a single-dose oral administration (Fig. 2) showed that the test formulation elicited a similar profile and pharmacokinetic parameters as the reference formulation (Table 2); and these values stayed inside the confidence interval. The $90 \%$ CIs for the test/reference LSM (least squares mean) ratios of the log-transformed data for $C_{\max }, A U C_{0-t}, A U C_{0-\infty}$, and $T_{\max }$ were $95.37,95.59,96.34$ and 105.98 , respectively. No treatment, study period, or sequence effects were found on ANOVA of mentioned parameters. The intrasubject variabilities $\left(\mathrm{CVs}^{2}\right)$ for the log-transformed data of $C_{\max }, A U C_{0-t}, A U C_{0-\infty}$ and $T_{\max }$ were $18,16.6,18.3$ and 33.2 , respectively. The difference in $T_{\max }$ values between the two products was not statistically significant according to the Anderson Hauck Procedure test $(p=0.0151)$.

\section{Discussion}

Bioequivalent preparations are considered to be therapeutically equivalent if the drug products exhibit comparable rate and extent of absorption when administered in the same dosage and under similar experimental conditions [9]. Bioequivalence is accepted by international agencies, as well as the Chilean National Regularity Authority, if the 90\% confidence intervals are achieved for the primary pharmacokinetic parameters $A U C_{0-\infty}, A U C_{0-t}$ and $C_{\max }$, allowing an acceptance range of $80 \sim 125 \%$ [9]. ANOVA (analysis of variance) showed no significant differences between preparations on any of the pharmacokinetic parameters for both hydrochlorothiazide formulations. For $C_{\max }, A U C_{0-t}$ and $A U C_{0-\infty}$, ANOVA for confidence level 90.00 and power at 20\% were $0.999737,0.999932$ and 0.999678 , respectively. Also, no statistically significant period effect was detected. The intrasubject variability was characterized by the coefficient of variation derived from ANOVA. It is important to note that in our study, the number of volunteers was greater than calculated from intra-individual variability. Both formulations presented good tolerability. Four non-serious adverse events were reported, all of which were considered to be mild. In the post study safety analyses, no alteration in clinical parameters was observed, and the volunteers received a medical discharge by the medical team.

\section{Conclusions}

The purpose of this study was to assess the bioequivalence of the test tablets compared to the reference tablets, both containing $50 \mathrm{mg}$ hydrochlorothiazide. The extent of absorption, as reflected by the values for $A U C_{0-\infty}$ and $A U C_{0-t}$, and the rate of absorption as reflected by $C_{\max }$ and $T_{\max }$ values, demonstrate bioequivalence since both extremes lie within the required $90 \%$ confidence intervals $80 \sim 125 \%$. Therefore, it can be concluded that both hydrochlorothiazide preparations are bioequivalent and are therapeutically equivalent and exchangeable in the clinical setting. This is an important advance to provide to general population an equitative access to drugs with high quality and guaranteed therapeutic effects., and can place us the basis to build the initial knowledge to study, in the future, the pharmacogenetic profile of this 
population, and the impact of metabolic capacities into the adverse reactions associated to this type of drugs or similar.

Certainly thiazide-like diuretics are less expensive antihypertensive agents and therefore are favored in terms of cost minimization. In terms of cost hydrochlorothiazide test turns out to be almost five times cheaper than the reference drug, becoming a real contribution to antihypertensive therapy without compromising quality.

\section{Acknowledgments}

We are grateful of Laurie Aguayo, by her careful correction of this work. The study was sponsored by Laboratorio Chile, Teva, and conducted by Centro de Investigaciones Avanzadas en Biomedicina-UdeC (CIAB2013-01-HCT), Chile.

\section{References}

[1] Brunton, L., Chabner, B., and Knollman, B. 2011. Goodman and Gilman's: The Pharmacological Basis of Therapeutics. 12th ed. New York: McGraw-Hill.

[2] Materson, B. J. 1983. "Insights into Intrarenal Sites and Mechanisms of Action of Diuretic Agents." Am. Heart J. 106: 188-208.

[3] Lacy, C. F., Armstrong, L. L., and Goldman, M. P. 2005. Lexi-Comp's Drug Information Handbook International: With Canadian and International Drug Monographs. 13ed. Hudson: Lexi-Comp.
[4] Beermann, B., Groschinsky-Grind, M., and Rosén, A. 1976. "Absorption, Metabolism, and Excretion of Hydrochlorothiazide.” Clin. Pharmacol. Ther. 19 (5): 531-7.

[5] Salvetti, A., and Ghiadoni, L. 2006. "Thiazide Diuretics in the Treatment of Hypertension: An Update." J. Am. Soc. Nephrol. 17 (4 Suppl 2): S25-9.

[6] Rita, B., and Akhilesh, T. 2015. "Importance of Bioequivalence Studies for Enhancing Pharmacokinetic Parameters." Res. Rev. J. Pharm. Nanotech. 3: 89-96.

[7] Chow, S. C., and Liu, J. P. 2009. Design and Analysis of Bioavailability and Bioequivalence Studies. 3rd ed. Boca Raton: Chapman \& Hall/CRC.

[8] Koytchev, R., Ozalp, Y., Erenmemisoglu, A., Van der Meer, M. J., and Alpan, R. S. 2004. "Effect of the Combination of Lisinopril and Hydrochlorothiazide on the Bioequivalence of Tablet Formulations." Arzneimittelforschung 54 (9A): 605-10.

[9] Niopas, I., Daftsios, A. C., and Nikolaidis, N. 2004. "Evaluation of the Bioequivalence of Two Tablet Formulations of Enalapril/Hydrochlorothiazide after Single Oral Administration to Healthy Volunteers." Arzneimittelforschung 54 (3): 160-5.

[10] WMA (World Medical Association). 2002. WMA Declaration of Helsinki Ethical Principles for Medical Research Involving Human Subjects. 53rd ed. Washington, D.C.: World Medical Association.

[11] FDA (US Food and Drug Administration). 2003. "Guidance for Industry Bioavailability and Bioequivalence Studies for Orally Administered Drug Products." In US Department of Health and Human Services; Center for Drug Evaluation and Research, edited by Rockville, M. D. Silver Spring: US Food and Drug Administration. 